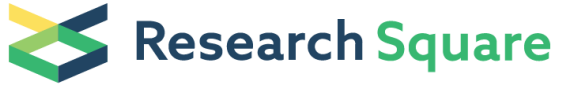 \\ Preprints are preliminary reports that have not undergone peer review. \\ They should not be considered conclusive, used to inform clinical practice, or referenced by the media as validated information.
}

\section{Identification of the Bcl-2 and Bax Homologs From the Tick Rhipicephalus Haemaphysaloides and Their Function in the Degeneration of Tick Salivary Glands}

\section{Shanming Hu}

Shanghai Veterinary Research Institute Chinese Academy of Agricultural Sciences

\section{Yanan Wang}

Shanghai Veterinary Research Institute Chinese Academy of Agricultural Sciences

\section{Zhengmao Xu}

Shanghai Veterinary Research Institute Chinese Academy of Agricultural Sciences

\section{Yongzhi Zhou}

Shanghai Veterinary Research Institute Chinese Academy of Agricultural Sciences Jie Cao

Shanghai Veterinary Research Institute Chinese Academy of Agricultural Sciences Houshuang Zhang

Shanghai Veterinary Research Institute Chinese Academy of Agricultural Sciences Jinlin Zhou ( $\square$ jinlinzhou@shvri.ac.cn )

Shanghai Veterinary Research Institute https://orcid.org/0000-0002-3383-1084

\section{Research}

Keywords: Salivary gland degeneration, Apoptosis, Bcl-2, Bax

Posted Date: January 28th, 2021

DOI: https://doi.org/10.21203/rs.3.rs-154051/v1

License: (9) (i) This work is licensed under a Creative Commons Attribution 4.0 International License. Read Full License

Version of Record: A version of this preprint was published at Parasites \& Vectors on August 4th, 2021. See the published version at https://doi.org/10.1186/s13071-021-04879-z. 


\section{Abstract}

\section{Background}

The salivary gland of female ticks degenerates rapidly by apoptosis and autophagy after feeding. Bcl-2 family proteins play an important role in the apoptosis pathways but the functions of these proteins in ticks are unclear. We studied Bcl-2 and Bax homologs from the tick Rhipicephalus haemaphysaloides and determined their functions in the degeneration of the salivary glands.

\section{Methods}

The full-length cDNA of the RhBcl-2 and RhBax genes were obtained by transcriptome analysis and $\mathrm{RhBcl}-2$ and $\mathrm{RhBax}$ were expressed in E. coli. Two molecules containing conserved $\mathrm{BH}$ (Bcl-2 family homology) domains were identified and named RhBcl-2 and RhBax. After protein purification and mouse immunizations, specific polyclonal antibodies (PcAb) were created in response to the recombinant protein. Reverse transcription quantitative PCR (RT-qPCR) and western blot were used to detect the existence of RhBcl-2 and RhBax in ticks. TUNEL assays were used to determine the apoptosis level in the salivary glands at different feeding times after gene silencing. The interactions between RhBcl-2 and RhBax were identified by co-transfection and GST pull-down assays. The RT-qPCR assay demonstrated that the transcription of RhBax genes increased significantly during the engorged periods of the tick developmental stages (engorged larval, nymph, and adult female ticks).

Results

Transcriptional levels of RhBcl-2 and RhBax in the salivary glands increased more significantly than other tissues post engorgement. RhBcl-2 treatment significantly restrained tick feeding. In contrast, RhBax interference had no effect on tick feeding. TUNEL staining showed that apoptosis levels were significantly reduced after interfering with $\mathrm{RhBcl}-2$ expression. Co-transfection and GST pull-down assays showed that RhBcl-2 and RhBax could combine with each other, but failed to combine without the $\mathrm{BH} 3$ domain.

Conclusions

This study identified the roles of RhBcl-2 and RhBax in tick salivary gland degeneration and clarifies their interactions.

\section{Introduction}

Ticks are obligatory blood-feeding arthropods and vectors of many pathogens [1]. Ticks usually stay attached to their hosts and feed for several days or even weeks [2]. The salivary glands of ticks mediate diverse functions that ensure tick survival [3]. Tick-borne pathogens (TBP) are transmitted to the host by the saliva used during tick feeding [4]. 
The salivary glands of female ticks degenerate rapidly within four days after engorgement [5]. Salivary gland degeneration begins at the early stage of rapid feeding and may be caused by apoptosis and autophagy related to the increase of ecdysone in the hemolymph [6, 7]. During degeneration, the granular acini undergo DNA fragmentation and caspase enzyme activity increases $[8,9]$. According to the comprehensive analysis of the global protein changes, some proteins associated with apoptosis and autophagy changed and some proteins linked to the degradation of DNA and proteins were consistently upregulated [10]. However, the mechanism of these effects is unknown.

Apoptosis is an important part of programmed cell death (PCD). Apoptosis is programmed to transmit death induction signals and carry out biochemical processes of cell decomposition by a group of specific interacting proteins [11]. For embryonic development, tissue homeostasis, and organism stability in multicellular organisms, apoptosis is indispensable and is a normal physiological process [12]. During apoptosis, a decrease in cell volume, cytoplasmic condensation, mitochondrial membrane permeabilization, DNA fragmentation, and chromatin condensation followed by nuclear fragmentation and cytoplasmic membrane blebbing take place, which leads to complete cell division. In the late stage of apoptosis, apoptotic bodies are produced due to cell fragmentation [13].

Apoptosis is caused by sequential activation of cysteine proteases of the caspase family in two distinct, but converging pathways [14]. The extrinsic pathway, also known as the death receptor pathway, activates Caspase- 8 when the "death receptor" Fas binds to its ligand FasL, and then recruits FADD proteins and caspase-8 enzymes in the cytoplasm to form a death complex DISC [15]. The intrinsic pathway (also termed the mitochondrial pathway) can be triggered by the pro-apoptotic proteins of the $\mathrm{Bcl}-2$ family. These cause mitochondrial membrane permeabilization to release mitochondrial cytochrome $\mathrm{c}$ into the cytoplasm, further activating caspases to induce apoptosis [16]. These initiator caspases can cleave and activate the effector caspases (Caspase- 3 , Caspase- 6 , and Caspase-7) that mediate cellular demolition by cleaving multiple critical cellular proteins [17].

The Bcl-2 (B-cell lymphoma-2) protein family is involved in apoptosis via the intrinsic pathway. Bcl-2 was first discovered via the chromosome translocation that hallmarks human follicular lymphoma, and it was subsequently found to promote cell survival as an inhibitory apoptosis protein of the Bcl-2 protein family [18]. Its homologs Bcl-X, Bcl-w, Mcl-1, and A1 were also found to inhibit apoptosis, but Bax, Bak, and Bok promoted apoptosis. $\mathrm{BH}$ (Bcl-2 homology) domain is a vital functional domain for the interaction of the Bcl-2 family proteins. The BH3 domain was a key for Bax, Bak, and Bok to promote apoptosis [19]. Bim, Bid, Bad, Puma, and Noxa only had the BH3 domain, so they were termed only-BH3 proteins.

The $\mathrm{Bcl}-2$ family proteins regulate apoptosis mainly by regulating mitochondrial membrane permeability, working by themselves or in combination with other proteins. For example, Bax forms oligomers on the mitochondrial membrane that perturb membrane integrity and promote apoptosis, while Bcl-2 and Bcl-xL inhibit their effects by binding to Bax to form heterodimers [20,21]. BH3-only protein can play a direct or indirect pro-apoptotic function. It can directly bind and activate Bax and Bak proteins to promote 
apoptosis, and it can indirectly promote the expression of Bax and Bak proteins to cause apoptosis by inhibiting the action of the anti-proteins $[22,23]$.

Bcl-2 family proteins exist in both mammalian and non-mammalian species. The nematode Caenorhabditis elegans has two members of the Bcl-2 family, EGL-1 and CED-9, that regulate cell death [24]. Two Bcl-2 family proteins which have high homology with Bok found in Drosophila, Debcl and Buffy [6]. Using RNA interference, Ayllon [25] injected Bcl-2 dsRNA into Ixodes scapularis and showed feeding disruption. This indicated that Bcl-2 plays an important role in the intrinsic apoptosis of ticks. However, the mechanism by which Bcl-2 family proteins regulate apoptosis in ticks is unknown. In this study, we identified Bcl-2 and Bax from the tick Rhipicephalus haemaphysaloides and investigated their functions in the degeneration of tick salivary glands at different feeding times.

\section{Material And Methods}

\subsection{Tick Feeding and tissue collection}

Adult Rhipicephalus haemaphysaloides Supino 1897 (Ixodida: Ixodidae) were collected from Wuhan in Hubei Province, China. The ticks were fed on the ears of New Zealand white rabbits (SLAC, Shanghai Institutes for Biological Science, CAS), and maintained in artificial climate incubators of the Shanghai Veterinary Research Institute [26]. Rabbits and mice were maintained following the approved guidelines of the Institutional Animal Care and Use Committee of the Shanghai Veterinary Research Institute (IACUC approval number: SHVRI-SZ-20190726-02; SHVRI-SZ-20191024-01). Tick tissues were rapidly dissected, washed with phosphate-buffered saline (PBS, PH 7.4, with $0.14 \mathrm{M} \mathrm{NaCl}$ and $0.0027 \mathrm{M} \mathrm{KCl}, 0.01 \mathrm{M}$ phosphate buffer; Gibco, Life Technologies), and placed in PBS or TRIzol (Invitrogen, Carlsbad, CA, USA) reagent at $-80^{\circ} \mathrm{C}$.

\subsection{RNA extraction and cDNA synthesis}

RNA was derived from ticks dissected at different development stages (eggs, unfed and fed larvae, nymphs, and adults) and different tissues (salivary glands, midguts, and ovaries of female ticks), and preserved in TRIzol reagent. The HiScript ${ }^{\circledR}$ III RT SuperMix for qPCR (+ gDNA wiper) kit (Vazyme Biotech, Nanjing, China) was used, according to manufacturer protocol, to synthesis cDNA.

\subsection{Cloning, sequence analysis, and expression of RhBcl-2 and RhBax}

$\mathrm{RhBcl}-2$ and $\mathrm{RhBax}$ primers were designed based on a comparison of the salivary gland transcriptomes of starved and engorged $R$. haemaphysaloides [27, 28]. A BLAST analysis of the translation products deduced from the open reading frames (ORFs) was performed. SignalP 4.1

(http://www.cbs.dtu.dk/services/SignalP/) [29] and ExPASy (http://web.expasy.org/compute pi/) [30] were used for the Signal peptide analysis and isoelectric point $(\mathrm{PI})$ prediction. We aligned RhBcl-2 and $\mathrm{RhBax}$ with the Bcl-2 and Bax protein sequences of other species using Genetyx ver. 6 (GENETYX, Tokyo, 
Japan). For phylogenetic analysis, the alignment of the sequences was performed using the MUSCLE algorithm [31] and inferred using the Maximum likelihood method with the default settings in MEGAX software [32]. Bootstrap support values were estimated using 500 bootstrap replicates [28].

\subsection{RT-qPCR analyses}

The expression levels of RhBcl-2 and RhBax were examined in ticks from different development stages (eggs, unfed and fed larvae, nymphs, and adults) and different tissues (salivary glands, midguts, and ovaries of female ticks). After RhBcl-2 and RhBax primers (Table S1) designed using Primer Premier 5, the cDNAs of the above stages and tissues were analyzed by RT-qPCRs which were conducted using ChamQ Universal SYBR qPCR Master Mix (Vazyme) green and gene-specific (Table S1) primers with a QuantStudio 5 PCR System (Applied Biosystems, Austin, TX, USA). The RT-qPCR process was $95^{\circ} \mathrm{C}$ for 30 $\mathrm{s}$, followed by 40 cycles of $95^{\circ} \mathrm{C}$ for $5 \mathrm{~s}$ and $60^{\circ} \mathrm{C}$ for $30 \mathrm{~s}$, followed by analysis of the melting curve. All samples were analyzed in triplicate. The data used elongation factor-1(ELF1A, Genbank accession no. AB836665) as an internal control [31], and this was used to analyze the relative gene expression in each sample by the $2^{-\triangle \mathrm{Ct}}$ method $[32,33]$.

\subsection{Expression of recombinant $\mathrm{RhBcl}-2$ or $\mathrm{RhBax}$ and antibody production}

Specific RhBcl-2 and RhBax primers (Table S2) were designed in a pET-30a or PGEX-4T-1 vector. The $\mathrm{RhBcl}-2$ and RhBax amplified-PCR products were purified and digested with $\mathrm{BamH}, \mathrm{EcoRl}$, or Xhol (New England Biolabs, USA) and ligated into pET-28a or PGEX-4T-1 (Invitrogen) using In-Fusion HD Cloning Kits (Takara Clontech, Mountain View, CA, USA) [28]. These recombinants were transformed and expressed in Escherichia coli (E. coli) BL21 (DE3) strain (TIANGEN, Beijing, China). The strains were grown until the $\mathrm{OD}_{600}$ reached 0.8 at $37^{\circ} \mathrm{C}$. After isopropyl $\beta$-D thiogalacto pyranoside (IPTG) was added to $1 \mathrm{mM}$, the protein expression was induced at $25^{\circ} \mathrm{C}$ for $20 \mathrm{~h}$. The recombinant, containing His-tagged protein, was purified by affinity chromatography using Ni-NTA His resin (Thermo Fisher Scientific, Waltham, MA, USA) by gravity. The recombinant containing the GST-tagged protein was purified by affinity chromatography using resin and gravity.

\subsection{GST-Pull Down of RhBcl-2 and RhBax}

To confirm whether RhBcl-2 interacts with RhBax, 1 mM IPTG induced expression for $8 \mathrm{~h}$ of pET-30aRhBax, PGEX-4T-RhBcl-2, and PGEX-4T-1 recombinant E. coli BL21. The supernatant was separated using GST agarose (Merck, Darmstadt, Germany) according to manufacturer instructions. Anti-GST antibody (Proteintech, 215 Rosemont, IL, USA) and goat anti-mouse secondary antibody (Proteintech) were used to confirm that GST/GST-RhBcl-2 was separated successfully, and anti-His antibody (Proteintech) was used to detect the target protein.

\subsection{Cells and Transient Co-transfection assays}

HEK 293 cells were maintained in Dulbecco's Modified Eagle Medium (DMEM, Gibco), supplemented with $8 \%$ heat-inactivated fetal bovine serum (Biological Industries, Kibbutz Beit Haemek, Israel) and 1\% 
penicillin (Gibco) at $37^{\circ} \mathrm{C}[28]$.

The full-length ORF of RhBcl-2 was inserted into the p3×Flag-CMV-14 vector (MiaoLing Plasmid Sharing Platform, Wuhan, China) with Flag tag at the N-terminal with gene specific primers (Table S2). RhBax was inserted into the pcmv-HA vector using same method. The fragment of RhBax deleted $\mathrm{BH} 3$ domain (Rhbax- $\triangle \mathrm{BH} 3$ ) and $\mathrm{BH} 1$ domain (Rhbax- $\triangle \mathrm{BH} 1$ ) was amplified by splicing by overlap extension (SOE) of recombinant PCR with specific primers (Table S3), and they were inserted into the pcmv-HA vector [34]. Transfection, using Lipofectamine ${ }^{\mathrm{TM}} 3000$ Transfection Reagent (Invitrogen), was performed according to manufacturer protocol with a DNA to Lipofectamine ratio of 1: $2 \mathrm{w} / \mathrm{v}$. The HEK 293 cells were transformed with $2.5 \mu \mathrm{g} /$ well of plasmid or co-transfected with the equivalent amount of two different plasmids in 6-well plates [28].

\subsection{Antibody generation and western blotting}

Recombinant $\mathrm{RhBcl}-2$ and $\mathrm{RhBax}$ were expressed and purified according to previously described protocols [35]. Polyclonal antibodies (pcAb) against recombinant RhBcl-2 and RhBax were developed in mice. We tested the specific recognition of the recombinant protein by the antibodies using western blots. We predicted the epitopes of RhBcl-2 and RhBax online (http://www.iedb.org/), and synthesized Keyhole limpet hemocyanin (KLH) [36] coupled polypeptides based on the epitope amino acid sequences (RhBcl2: GLQWNTCPPLPRPSK, RhBax: STPTHEETREE). Polypeptides were dissolved in PBS and Freund's adjuvant (complete and incomplete; Invitrogen). Equal volumes of these solutions were then emulsified together and injected into six to eight weeks old BALC/c mice provided by the Shanghai Laboratory Animals Center (Shanghai Institutes for Biological Science, Chinese Academy of Sciences, Shanghai, China). The mixtures were injected three times at two weeks intervals. The sera were collected on the seventh day after the third immunization.

Total proteins of different development stage ticks were extracted using Tris-buffered saline (TBS; $10 \mathrm{mM}$ Tris- $\mathrm{HCl}, \mathrm{pH}$ 7.5; $150 \mathrm{mM} \mathrm{NaCl}$ with $1 \mathrm{mM}$ phenylmethanesulfonyl fluoride). A Bradford Protein Assay Kit (Beyotime Tiangen) was used to determine the protein concentration. Proteins were separated by $10 \%$ sodium dodecyl sulphate polyacrylamide gel electrophoresis (SDS-PAGE, $12 \%$, Genescript, Nanjing, China) and transferred onto polyvinylidene difluoride (PDVF) membranes. After blocking, the proteins were incubated at $4^{\circ} \mathrm{C}$ overnight with primary antibodies. The sera anti RhBcl-2 and RhBax were used to detect RhBcl-2 and RhBax in protein extracts, and anti-tubulin (1:1000, Proteintech, Rosemont, IL, USA) primary antibody was used as a constitutive control to normalize the signal from the target protein. The membranes were rinsed five times in tris-buffered saline with Tween-20 (TBST) and incubated with a secondary antibody (goat anti-mouse, 1:5000, Cell Signaling Technology, USA) for $1 \mathrm{~h}$ at room temperature. The membranes were rinsed five times by TBST. Images were captured by ChemiDoc Touch (Bio-rad, Hercules, CA, USA) [28].

\subsection{RNAi of RhBcl-2 or RhBax}

The RNAi experiments were designed against RhBcl-2 and RhBax genes. For the design of RNAi primers, RhBcl-2 and RhBax sequences were screened by Primer Premier 5. Specific primers (Table S4) containing 
the T7 polymerase promoter sequence were used for PCR amplification. The amplicons were then purified to obtain templates for double-stranded RNA synthesis using the T7 RiboMAX Express RNAi system (Promega, Madison, WI, USA). The unrelated gene Luciferase dsRNA was synthesized using the same methods described previously and used as the negative control. Unfed female ticks ( $N=20$ females per group) were microinjected with approximately $1 \mu \mathrm{g}$ of dsRNA. Control ticks were injected with unrelated Luciferase dsRNA. After dsRNA injection, female ticks were held in a humidity chamber for 1 day after which they were allowed to feed on rabbit ears. The biological parameters analyzed were attachment rate at $24 \mathrm{~h}$, number of engorged ticks, death rate, and weight. RNA was used to characterize gene knockdown by real-time RT-PCR.

We interfered with the salivary gland using dsRNA in vitro. The salivary gland of female ticks fed on rabbit for 5 days was dissected and placed into complete L15 medium with $1 \%$ penicillin-streptomycin [37]. Each well received $5 \mu \mathrm{g}$ of dsRNA and incubated at $27^{\circ} \mathrm{C}$ with no $\mathrm{CO}_{2}$ for $48 \mathrm{~h}$. The control group received unrelated Luciferase dsRNA. Each treatment group included the salivary glands from 10 ticks. After incubation, a pair of the salivary glands in each group was fixed in $4 \%$ paraformaldehyde for TUNEL staining or fixed in $2.5 \%$ glutaraldehyde for at least $24 \mathrm{~h}$ at $4^{\circ} \mathrm{C}$ for TEM. The remaining salivary glands were used for western blots and RNA.

\subsection{TUNEL staining}

Salivary glands were fixed in $4 \%$ formalin and paraffin-embedded. Sections of salivary glands were mounted on microscope slides. Tissue sections were then deparaffinized, washed in $100 \%$ ethanol, and rehydrated. Samples were washed with PBS. After antigen retrieval with $0.1 \%$ Triton X-100, the tissues were incubated for $1 \mathrm{~h}$ with 1:9 TdT mixed with fluorescent-labeled dUTP at $37^{\circ} \mathrm{C}$, following the instructions of the Roche In Situ Cell Death Detection Kit, POD (Roche, Mannheim, Germany). After washing the Sect. 2-3 times with PBS, the sections were stained with $1 \mu \mathrm{g} / \mathrm{mL}$ 4', 6'-diamidino-2phenylindole (DAPI, Invitrogen) in distilled $\mathrm{H}_{2} \mathrm{O}$ for 20 min [28]. After washing, the sections were mounted using Lab Vision ${ }^{\text {TM }}$ PermaFluor ${ }^{\text {TM }}$ (Invitrogen) medium under glass coverslips, then viewed and photographed on a Pannoramic DESK Digital Slide Scanner (3D HISTECH, Budapest, Hungary) [28].

\subsection{Data analysis}

All statistical analyses were performed using GraphPad Prism 6.0 software (Graph Pad Software Inc., San Diego, CA, USA). Mean \pm standard error (SEM) values were calculated for three separate experiments, and two-tailed Student's $t$ tests were used to identify significant differences between groups. $P<0.05$ was considered to be statistically significant.

\section{Results}

\subsection{Sequencing analysis of $\mathrm{RhBcl}-2$ and $\mathrm{RhBax}$}


We used nucleic acid sequences obtained from RNAsEq. Specific cloning primers were designed according to the predicted sequences of $R$. haemaphysaloides $\mathrm{Bcl}-2$ and $\mathrm{Bax}$ (Table S2). The ORF regions of $\mathrm{Bcl}-2$ and Bax genes were cloned from the CDNA of fully engorged $R$. haemaphysaloides salivary glands and named RhBcl-2 and RhBax. RhBcl-2 ORF has 576 bp and encodes a protein of 192 amino acid residues with a deduced molecular weight (MW) and theoretical isoelectric point (PI) of $21.4 \mathrm{kDa}$ and 4.87, respectively. RhBax ORF has 648 bp and encodes a protein with 216 amino acid residues. It has a deduced $\mathrm{MW}$ of $24.6 \mathrm{kDa}$ and a $\mathrm{PI}$ of 5.28 . RhBcl-2 had the conserved $\mathrm{BH} 1, \mathrm{BH} 2, \mathrm{BH} 3$, and $\mathrm{BH} 4$ domains, but RhBax had the conserved $\mathrm{BH} 1$ and $\mathrm{BH} 3$ domains.

\subsection{Transcription and translation of $\mathrm{RhBcl}-2$ and $\mathrm{RhBax}$ profiles in different stages, tissues, and feeding periods}

The cDNA of eggs, larvae (unfed and engorged), and nymphs (unfed and engorged) were subjected to RTqPCR to evaluate the expression profiles of $\mathrm{RhBcl}-2$ and $\mathrm{RhBax}$ genes during the developmental stages. The cDNA of unfed adults (females and males), fed adults (females and males), and engorged female ticks were used to determine the sex-specific profile of $\mathrm{RhBcl}-2$ and $\mathrm{RhBax}$ genes. The transcription level of RhBax increased after adult female feeding.

After microdissection, the cDNA of salivary glands, ovary, and midgut of adult female ticks at different feeding times were analyzed for RhBcl-2 and RhBax gene expression profiles. RhBcl-2 and RhBax genes were expressed in different tissues during tick feeding (including the early-feeding period, the fast-feeding period, and the end of the feeding period). However, RhBcl-2 and RhBax had the same expression trends in different organs.

Western blot analysis used anti-sera of RhBcl-2 and RhBax. RhBax was detected during the fast-feeding time (fed $5 \mathrm{~d}$ to $7 \mathrm{~d}$ ) to post engorgement and RhBcl-2 was detected at all stages.

\subsection{Expression of RhBcl-2 and RhBax}

The coding sequences of RhBcl-2 and RhBax were cloned into prokaryotic expression vectors (pET-28a and pGEX-4T-1) to produce recombinant RhBcl-2 and RhBax. All of the recombinant proteins were expressed as inclusion bodies in E. coli.

After solubilization and purification, His-RhBax (molecular weight: 38 kDa) and GST-RhBcl-2 (molecular weight: $\sim 50 \mathrm{kDa}$ ) were obtained, respectively. The MWs of $\mathrm{RhBcl}-2$ were likely the corresponding mammalian Bcl-2 molecules. Specific polyclonal antibodies (PcAb) were created in response to the recombinant protein. Western blot analysis revealed that the two sera were able to identify the full recombinant proteins, and the fragment sizes were consistent with those observed by SDS-PAGE.

\subsection{RhBcl-2 binds with RhBax by BH3 domain}

To verify that RhBcl-2 interacts with RhBax, an in-vitro GST-Pull down assay was used to confirm the interaction between His-RhBax and GST-RhBcl-2. For this experiment, two recombinant proteins with either a GST-tag or His-tag, respectively (His-RhBax and GST-RhBcl-2), were produced from E. coli BL21. 
Immunoblotting showed that the GST and GST-RhBcl-2 were successfully pulled down with GST beads and that the GST-RhBcl-2 interacted directly with His-RhBax. GST as a negative control confirmed that the interaction of GST-RhBcl-2 and His-RhBax was specific.

Co-transfection of HEK 293 cells with plasmids containing RhBcl-2 and RhBax genes resulted in coexpression of the proteins. The results also showed that $\mathrm{RhBcl}-2$ and $\mathrm{RhBax}$ could interact with each other, but failed to combine without the BH3 domain (Fig. 8).

\subsection{RNAi assay of RhBcl-2 and RhBax}

To identify the function of $\mathrm{RhBcl}-2$ and $\mathrm{RhBax}$, the genes of $\mathrm{RhBcl}-2$ and $\mathrm{RhBax}$ were in vivo targets of RNA interference. The RT-qPCR analysis of ticks showed decreased levels of RhBcl-2 and RhBax in RhBcl2-dsRNA and RhBax-dsRNA injected groups compared to luciferase-dsRNA injected controls (RhRhBcl-2: $\left.\mathrm{t}_{(3)}=3.967, P=0.0166 ; \operatorname{RhBax} \mathrm{t}_{(3)}=4.153, P=0.0142\right)$. Compared to the control groups, ticks injected with RhBax-dsRNA sere similar to the control groups, but ticks injected with RhBcl-2-dsRNA were significantly different. However, there was no significant difference in the attachment rate between the experiment and control groups.

TUNEL assays showed that salivary gland interference by RhBcl-2 dsRNA increased apoptosis levels, but RhBax was similar to the control.

\section{Discussion}

\section{Discussion}

$R$. haemaphysaloides is a three-host hard tick widely distributed in China. It is a carrier of several human pathogens [38, 39, 40]. The salivary glands of female ticks undergo degeneration after engorgement[3]. Apoptosis plays a role in the process while caspase genes and apoptosis-related genes are upregulated [41]. We identified and characterized two $\mathrm{Bcl}-2$ family proteins (Bcl-2 and $\mathrm{Bax}$ ) in R. haemaphysaloides and found that they are involved in salivary gland degeneration.

The Bcl-2 family proteins are also found in insects such as Drosophila, Bombyx mori, and Anopheline mosquitoes, and are involved in growth and metamorphosis [24, 42, 43, 44]. Ayllon [25] found that Bcl-2 may play a key role in the intrinsic apoptosis of Ixodes scapularis, but other Bcl-2 family proteins and the apoptosis mechanism are unknown. We found that RhBcl-2 and RhBax are involved in salivary glands apoptosis degeneration in $R$. haemaphysaloides. Sequence analysis demonstrated that $\mathrm{RhBcl}-2$ and RhBax have conserved sequences characteristic of BH domains. The BH3 domain was a key for Bax, Bak, and Bok to promote apoptosis and combine anti-apoptosis proteins with pro-apoptosis proteins $[45,46]$. A GST pull-down assay verified that $\mathrm{RhBcl}-2$ and $\mathrm{RhBax}$ combined with each other. Co-transfection assays also showed that $\mathrm{RhBcl}-2$ and $\mathrm{RhBax}$ could interact with each other but did not combine without the $\mathrm{BH} 3$ domain.

Salivary gland degeneration is triggered after the completion of female tick feeding [47]. The RT-qPCR results showed that RhBcl-2 and RhBax had a high level of transcription in salivary glands during tick 
engorgement as determined by western blot. These data suggest that the roles of RhBcl-2 and RhBax are related to apoptosis, and degeneration, of the salivary glands.

RNAi was used to study the physiological roles of RhBcl-2 and RhBax. TUNEL staining was used to evaluate the rate of DNA fragmentation in degenerated tick salivary glands. Compared to the control group, the TUNEL positive staining rate increased significantly by interfering with $\mathrm{RhBcl}-2$. These results showed that $\mathrm{RhBcl}-2$ functions in preventing apoptosis during degeneration of the salivary glands. Bax is a pro-apoptosis protein in the Bcl-2 protein family [48]. However, interfering with RhBax had no significant effect compared to the control group. This suggests that other apoptosis pathways help regulate the apoptosis of the salivary glands.

$\mathrm{RhBcl}-2$ silencing produced the most significant phenotype alteration in ticks during blood feeding compared to RhBax and the control groups. After injection with dsRhBcl-2, the female ticks were unable to feed and engorge until they died. These data illustrated the importance of $\mathrm{RhBcl}-2$ during blood feeding. Bcl-2 inhibited Bax to protect cell survival, but had other affects $[49,50]$. Consequently, RhBcl-2 functioned to protect cell survival, promote normal organ functioning, and ensure that ticks grew in the stage of blood feeding. The function of the RhBax protein remains unclear.

In conclusion, two Bcl-2 family molecules were identified in ticks and the roles of $\mathrm{RhBcl}-2$ and $\mathrm{RhBax}$ in tick physiology were studied. These findings allowed for advanced studies of tick apoptosis. RhBcl-2 and RhBax, like Bcl-2 and Bax in mammals, combine with each other to regulate apoptosis in the tick salivary glands. The knockdown of RhBcl-2 in vivo inhibited tick growth and blood feeding and demonstrated that $\mathrm{RhBcl}-2$ has the potential to be a tick vaccine protein.

\section{Abbreviations}

TBPs, Tick-borne pathogens; PCD, Programmed cell death; PcAb, polyclonal antibodies; RT-qPCR, reverse transcription quantitative PCR; PBS, Phosphate-buffered saline; TBS; $10 \mathrm{mM}$ Tris- $\mathrm{HCl}, \mathrm{pH} 7.5 ; 150 \mathrm{mM}$ $\mathrm{NaCl}$ with $1 \mathrm{mM}$ phenylmethanesulfonyl fluoride; SDS-PAGE, Sodium dodecyl sulfate polyacrylamide gel electrophoresis; IPTG, Isopropyl $\beta$-D-1-Thiogalactopyranoside; DAPI, 4', 6'-diamidino-2-phenylindole; TUNEL, Terminal deoxynucleotidyl transferase dUTP Nick-End Labeling; BH, Bcl-2 homology

\section{Declarations}

\section{Ethics approval and consent to participate}

The treatment of rabbits and mice was approved by the Institutional Animal Care and Use Committee of the Shanghai Veterinary Research Institute (IACUC approval number: SHVRI-SZ-20190726-02; SHVRI-SZ20191024-01), and authorized by the Animal Ethical Committee of Shanghai Veterinary Research Institute.

\section{Consent for publication}


Not applicable.

\section{Availability of data and material}

The datasets supporting the conclusions of this article are uploaded as Additional files.

\section{Competing interests}

The authors declare that they have no competing interests.

\section{Funding}

This work was supported by a grant from "Central Public-interest Scientific Institution Basal Research Fund (No. Y2021 GH01-3)" and Strategic International Collaborative Research Project (JPJ008837) Promoted by the Ministry of Agriculture, Forestry and Fisheries, Japan.

\section{Authors' contributions}

SH conceived the study, performed the statistical analyses and participated in the dynamic detection of the target genes. SH drafted the manuscript. YW and ZX participated in the design of the study and the interpretation of the data. $\mathrm{YZ}, \mathrm{HZ}$ and $\mathrm{JC}$ participated in the experiments on animals. JZ participated in the design of the study and helped to draft the manuscript. All authors read and approved the final manuscript.

\section{Acknowledgments}

We thank LetPub (www.letpub.com) for its linguistic assistance during the preparation of this manuscript.

\section{References}

1. Jongejan F, Uilenberg G. The global importance of ticks. Parasitology. 2004;129:S3-S14;

2. Sonenshine DE. Biology of ticks. New York: Oxford University Press; 1991.

3. Simo L, Kazimirova M, Richardson J, Bonnet SI. The Essential Role of Tick Salivary Glands and Saliva in Tick Feeding and Pathogen Transmission. Front Cell Infect Microbiol. 2017;7:281;

4. Kazimirova M, Stibraniova I. Tick salivary compounds: their role in modulation of host defences and pathogen transmission. Front Cell Infect Microbiol. 2013;3:43;

5. Francischetti IM, Sa-Nunes A, Mans BJ, Santos IM, Ribeiro JM. The role of saliva in tick feeding. Front Biosci (Landmark Ed). 2009;14:2051-88;

6. Abdelwahid E, Rolland S, Teng X, Conradt B, Hardwick JM, White K. Mitochondrial involvement in cell death of non-mammalian eukaryotes. Biochim Biophys Acta. 2011;1813 4:597-607; 
7. Mao H, Kaufman WR. Profile of the ecdysteroid hormone and its receptor in the salivary gland of the adult female tick, Amblyomma hebraeum. Insect Biochem Mol Biol. 1999;29 1:33-42;

8. L'Amoreaux WJ, Junaid L, Trevidi S. Morphological evidence that salivary gland degeneration in the American dog tick, Dermacentor variabilis (Say), involves programmed cell death. Tissue Cell. 2003;35 2:95-9;

9. Freitas DR, Rosa RM, Moura DJ, Seitz AL, Colodel EM, Driemeier D, et al. Cell death during preoviposition period in Boophilus microplus tick. Vet Parasitol. 2007;144 3-4:321-7;

10. Wang H, Zhang X, Wang X, Zhang B, Wang M, Yang X, et al. Comprehensive Analysis of the Global Protein Changes That Occur During Salivary Gland Degeneration in Female Ixodid Ticks Haemaphysalis longicornis. Front Physiol. 2018;9:1943;

11. Maghsoudi N, Zakeri Z, Lockshin RA. Programmed cell death and apoptosis-where it came from and where it is going: from Elie Metchnikoff to the control of caspases. Exp Oncol. 2012;34 3:146-52;

12. Kerr JF, Wyllie AH, Currie AR. Apoptosis: a basic biological phenomenon with wide-ranging implications in tissue kinetics. Br J Cancer. 1972;26 4:239-57;

13. Serrano-Heras G, Diaz-Maroto I, Castro-Robles B, Carrion B, Perona-Moratalla AB, Gracia J, et al. Isolation and Quantification of Blood Apoptotic Bodies, a Non-invasive Tool to Evaluate Apoptosis in Patients with Ischemic Stroke and Neurodegenerative Diseases. Biol Proced Online. 2020;22:17;

14. Shi Y. Mechanical aspects of apoptosome assembly. Curr Opin Cell Biol. 2006;18 6:677-84;

15. Yin F, Zhou H, Fang Y, Li C, He Y, Yu L, et al. Astragaloside IV alleviates ischemia reperfusion-induced apoptosis by inhibiting the activation of key factors in death receptor pathway and mitochondrial pathway. J Ethnopharmacol. 2020;248:112319;

16. Santos LC, Vogel R, Chipuk JE, Birtwistle MR, Stolovitzky G, Meyer P. Mitochondrial origins of fractional control in regulated cell death. Nat Commun. 2019;10 1:1313;

17. Adams JM, Cory S. Bcl-2-regulated apoptosis: mechanism and therapeutic potential. Curr Opin Immunol. 2007;19 5:488-96;

18. Tsujimoto $\mathrm{Y}$, Cossman J, Jaffe E, Croce CM. Involvement of the bcl-2 gene in human follicular lymphoma. Science. 1985;228 4706:1440-3;

19. Hartman ML, Czyz M. BCL-w: apoptotic and non-apoptotic role in health and disease. Cell Death Dis. 2020;11 4:260;

20. Gross A, Jockel J, Wei MC, Korsmeyer SJ. Enforced dimerization of BAX results in its translocation, mitochondrial dysfunction and apoptosis. Embo J. 1998;17 14:3878-85;

21. Minn AJ, Swain RE, Ma A, Thompson CB. Recent progress on the regulation of apoptosis by Bcl-2 family members. Adv Immunol. 1998;70:245-79;

22. Strasser A. The role of BH3-only proteins in the immune system. Nat Rev Immunol. 2005;5 3:189-200;

23. Deng J, Carlson N, Takeyama K, Dal Cin P, Shipp M, Letai A. BH3 profiling identifies three distinct classes of apoptotic blocks to predict response to ABT-737 and conventional chemotherapeutic agents. Cancer Cell. 2007;12 2:171-85; 
24. Igaki T, Miura M. Role of Bcl-2 family members in invertebrates. Biochim Biophys Acta. 2004;1644 23:73-81;

25. Ayllon N, Villar M, Galindo RC, Kocan KM, Sima R, Lopez JA, et al. Systems biology of tissue-specific response to Anaplasma phagocytophilum reveals differentiated apoptosis in the tick vector Ixodes scapularis. PLoS Genet. 2015;11 3:e1005120;

26. Zhou J, Gong H, Zhou Y, Xuan X, Fujisaki K. Identification of a glycine-rich protein from the tick Rhipicephalus haemaphysaloides and evaluation of its vaccine potential against tick feeding. Parasitol Res. 2006;100 1:77-84;

27. Yu X, Gong H, Zhou Y, Zhang H, Cao J, Zhou J. Differential sialotranscriptomes of unfed and fed Rhipicephalus haemaphysaloides, with particular regard to differentially expressed genes of cysteine proteases. Parasit Vectors. 2015;8:597;

28. Wang Y, Hu S, Tuerdi M, Yu X, Zhang H, Zhou Y, et al. Initiator and executioner caspases in salivary gland apoptosis of Rhipicephalus haemaphysaloides. Parasit Vectors. 2020;13 1:288;

29. Cruz LM, Trefflich S, Weiss VA, Castro MAA. Protein Function Prediction. Methods Mol Biol. 2017; 1654:55-75;

30. Artimo P, Jonnalagedda M, Arnold K, Baratin D, Csardi G, de Castro E, et al. ExPASy: SIB bioinformatics resource portal. Nucleic Acids Res. 2012;40 Web Server issue:W597-603;

31. Nijhof AM, Balk JA, Postigo M, Jongejan F. Selection of reference genes for quantitative RT-PCR studies in Rhipicephalus (Boophilus) microplus and Rhipicephalus appendiculatus ticks and determination of the expression profile of Bm86. BMC Mol Biol. 2009;10:112;

32. Livak KJ, Schmittgen TD. Analysis of relative gene expression data using real-time quantitative PCR and the 2(-Delta Delta C(T)) Method. Methods. 2001;25 4:402-8;

33. Ginzinger DG. Gene quantification using real-time quantitative PCR: an emerging technology hits the mainstream. Exp Hematol. 2002;30 6:503-12;

34. Kim HS, Smithies O. Recombinant fragment assay for gene targetting based on the polymerase chain reaction. Nucleic Acids Res. 1988;16 18:8887-903;

35. Wang Y, Li Z, Zhou Y, Cao J, Zhang H, Gong H, et al. Specific histamine binding activity of a new lipocalin from Hyalomma asiaticum (Ixodidae) and therapeutic effects on allergic asthma in mice. Parasit Vectors. 2016;9 1:506;

36. Greenfield EA, DeCaprio J, Brahmandam M. Making Weak Antigens Strong: Cross-Linking Peptides to KLH with Maleimide. Cold Spring Harb Protoc. 2018;2018 10:pdb prot100016;

37. Grabowski JM, Tsetsarkin KA, Long D, Scott DP, Rosenke R, Schwan TG, et al. Flavivirus Infection of Ixodes scapularis (Black-Legged Tick) Ex Vivo Organotypic Cultures and Applications for Disease Control. MBio. 2017;8 4;

38. Chen Z, Yang X, Bu F, Yang X, Yang X, Liu J. Ticks (acari: ixodoidea: argasidae, ixodidae) of China. Exp Appl Acarol. 2010;51 4:393-404; 
39. Yu Z, Wang H, Wang T, Sun W, Yang X, Liu J. Tick-borne pathogens and the vector potential of ticks in China. Parasit Vectors. 2015;8:24;

40. Zhang J, Liu Q, Wang D, Li W, Beugnet F, Zhou J. Epidemiological survey of ticks and tick-borne pathogens in pet dogs in south-eastern China. Parasite. 2017;24:35;

41. Yu X, Zhou Y, Cao J, Zhang H, Gong H, Zhou J. Caspase-1 participates in apoptosis of salivary glands in Rhipicephalus haemaphysaloides. Parasit Vectors. 2017;10 1:225;

42. Brachmann CB, Jassim OW, Wachsmuth BD, Cagan RL. The Drosophila bcl-2 family member dBorg-1 functions in the apoptotic response to UV-irradiation. Curr Biol. 2000;10 9:547-50;

43. Colussi PA, Quinn LM, Huang DC, Coombe M, Read SH, Richardson H, et al. Debcl, a proapoptotic Bcl2 homologue, is a component of the Drosophila melanogaster cell death machinery. J Cell Biol. 2000;148 4:703-14;

44. Igaki T, Kanuka H, Inohara N, Sawamoto K, Nunez G, Okano H, et al. Drob-1, a Drosophila member of the Bcl-2/CED-9 family that promotes cell death. Proc Natl Acad Sci U S A. 2000;97 2:662-7;

45. Chou JJ, Li H, Salvesen GS, Yuan J, Wagner G. Solution structure of BID, an intracellular amplifier of apoptotic signaling. Cell. 1999;96 5:615-24;

46. McDonnell JM, Fushman D, Milliman CL, Korsmeyer SJ, Cowburn D. Solution structure of the proapoptotic molecule BID: a structural basis for apoptotic agonists and antagonists. Cell. 1999;96 5:625-34;

47. Scopinho Furquim KC, Bechara GH, Camargo Mathias MI. Death by apoptosis in salivary glands of females of the tick Rhipicephalus sanguineus (Latreille, 1806) (Acari: Ixodidae). Exp Parasitol. 2008;119 1:152-63;

48. Oltvai ZN, Milliman CL, Korsmeyer SJ. Bcl-2 heterodimerizes in vivo with a conserved homolog, Bax, that accelerates programmed cell death. Cell. 1993;74 4:609-19;

49. Brooks C, Dong Z. Regulation of mitochondrial morphological dynamics during apoptosis by Bcl-2 family proteins: a key in Bak? Cell Cycle. 2007;6 24:3043-7;

50. Bettaieb A, Dubrez-Daloz L, Launay S, Plenchette S, Rebe C, Cathelin S, et al. Bcl-2 proteins: targets and tools for chemosensitisation of tumor cells. Curr Med Chem Anticancer Agents. 2003;3 4:307-18;

\section{Tables}

Table 1 Effect of knocking-down RhBcl-2 and RhBax on tick feeding behavior.

\begin{tabular}{|llllll|}
\hline Test groups & $\begin{array}{l}\text { Attachment rate at } \\
24 \mathrm{~h}(\%)\end{array}$ & $\begin{array}{l}\text { Engorgement } \\
\text { rate (\%) }\end{array}$ & $\begin{array}{l}\text { Death } \\
\text { rate (\%) }\end{array}$ & $\begin{array}{l}\text { egg laying } \\
\text { rate (\%) }\end{array}$ & $\begin{array}{l}\text { Hatchability } \\
\text { rate (\%) }\end{array}$ \\
\hline dsLuciferase & $76.67 \%$ & $73.33 \%$ & $20.00 \%$ & $65.00 \%$ & $65.00 \%$ \\
\hline dsRhBcl-2 & $85.00 \%$ & $0.00 \%$ & $95.00 \%$ & $0.00 \%$ & $0.00 \%$ \\
\hline dsRhBax & $70.00 \%$ & $61.67 \%$ & $33.33 \%$ & $48.33 \%$ & $48.33 \%$ \\
\hline
\end{tabular}




\section{Figures}

(A)

RhBax

$\mathrm{H}$ : sapiens 1:MDGSGEQPRGGGPTSSEQIMKTGALLLQGFIQD---RAGR-MGGEAPELALDP--VPQDA 54

M: musculus 1:MDGSGEQLGSGGPTSSEQIMKTGAFLLQGFIQD---RAGR-MAGETPELTLEQ--PPQDA 54

R: haemaphysaloides 1:MSIPLPLLRST-PTHEETREE-GRVLLSSFVDYQVAQEGAAMDAGASSESIGDMVYSHTI 58

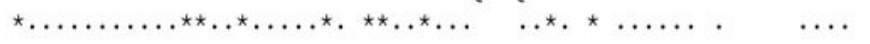

$\mathrm{H}$ : sapiens 55:STKKLS-------ECLKRIGDELDSNME-LQRMIAA------V--DTD--SP----REV 91

M: musculus 55:STKKLS------ECLRRIGDELDSNME-LQRMIAD------V--DTD--SP----REV 91

R: haemaphysaloides 59:DEEDIGTPHSRTEAEALHPVYRNMGRELRALADRFSQSRERIRVRQEADSLDLSSLTRDN 118

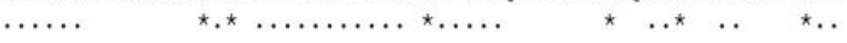

H: sapiens 92:FFRVAADMFSDGNFNWGRVVALFYFASKLVLKAL-CTKVPELIRTIMGWTLDFLRERLLG 150

M: musculus 92:FFRVAADMFADGNFNWGRVVALFYFASKLVLKAL-CTKVPELIRTIMGWTLDFLRERLLV 150

R: haemaphysaloides 119:LTTLMLELFEDG-FSRERLVTFFFFCSDLILKSVRCSVG-GLRWQVVAWVWAFLRDRVCC 176

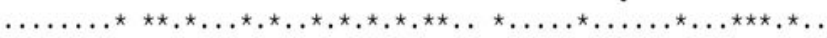

H: sapiens 151:WIQDQGGWDGLLSY-FGTPTWQTVTIFVAGVLTASLTIWKKMG 192

M: musculus 151:WIQDQGGWEGLLSY-FGTPTWQTVTIFVAGVLTASLTIWKKMG

R: haemaphysaloides 177:WVLQHGGWEAVLTNYLPKLAITAAG-FAVCVGVVFY-IWKNW- 216

RhBcl-2

$\mathrm{H}$ : sapiens 1:MAHAGRTGYDNREIVMKYIHYKLSQRGYEWDAGDVGAAPPGAAPAPGIFSSQPGHTPHPA 60

M: musculus 1:MAQAGRTGYDNREIVMKYIHYKLSQRGYEWDAGDADAAPLGAAPTPGIFSFQPESNPMPA 60

R: haemaphysaloides 1:MAVNEAEKTQVNELVNDFMRYQLNRRGLQWN-

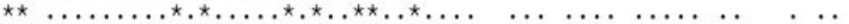

H: sapiens 61:ASRDPVARTS-PLQTPAAPGAAAGPALSPVPPVVHLTLRQAGDDFSRRYRRDFAEMSSQL 119 M: musculus 61:VHRDMAARTS-PLRPLVATA---GPALSPVPPVVHLTLRRAGDDFSRRYRRDFAEMSSQL 116 $\mathrm{R}$ : haemaphysaloides $32:-\cdots$

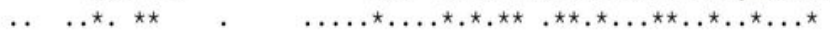

H: sapiens 120:HLTPFTARGRFATVVEELFRDGVNWGRIVAFFEFGGVMCVESVNREMSPLVDNIALWMTE 179 M: musculus 117:HLTPFTARGRFATVVEELFRDGVNWGRIVAFFEFGGVMCVESVNREMSPLVDNIALWMTE 176

R: haemaphysaloides 69:DMTPSVAQTAYTDVLNELFSEGITWARIVGAFAFSVELSALCVEKNWSELVDSIASWLSS 128

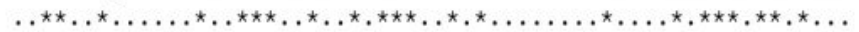

$\mathrm{H}$ : sapiens 180:YLNRHLHTWIODNGGWDAFVELYGPSMRPLFDF--SWLSLKTLLSL-ALVG-ACI-TLGA 234 M: musculus 177:YLNRHLHTWIQDNGGWDAFVELYGPSMRPLFDF--SWLSLKTLLSL-ALVG-ACI-TLGA 231

R: haemaphysaloides 129:YVCTRLLPWIKDHQGWDGLVV-FSEERDDTGAAESPWPIFKNVVCGVA-VTALGALTLGA 186

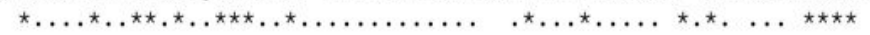

H: sapiens 235:YLGHK- 239

$\begin{array}{lll}\text { M: musculus 232:YLGHK- } & 236\end{array}$

R: haemaphysaloides 187:FLTSKS 192

.*. . *

(B)

\section{RhBax}

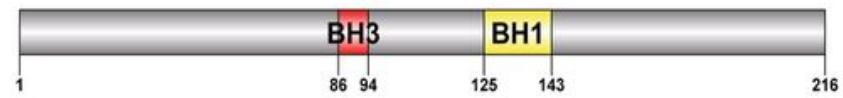

\section{RhBcl-2}

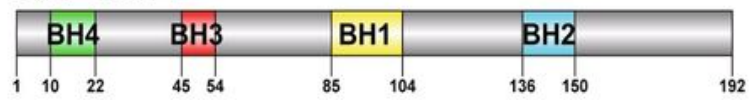

\section{Figure 1}


musculus Bcl-2: NP_803129.2; Homo sapiens Bax: NP_001278357.1; Mus musculus Bax: NP_031553.1; (B) Domain structure of RhBcl-2 and RhBax.

Figure 2

(A)

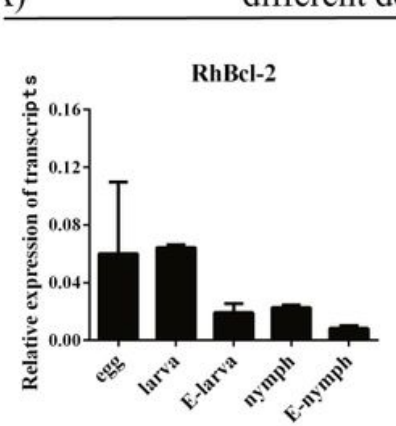

(B)

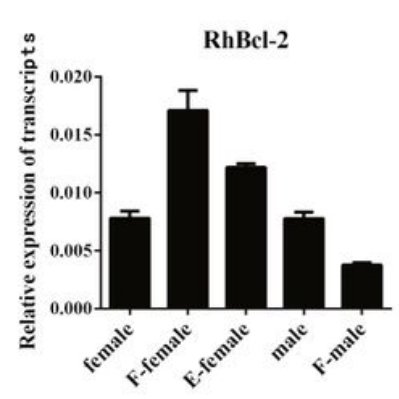

different sex

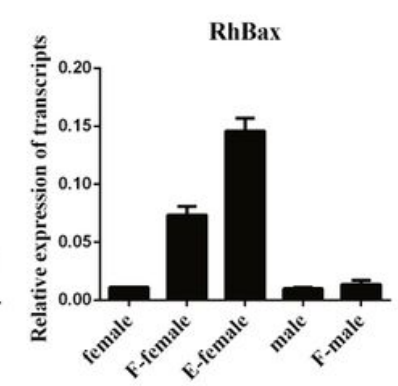

(C)

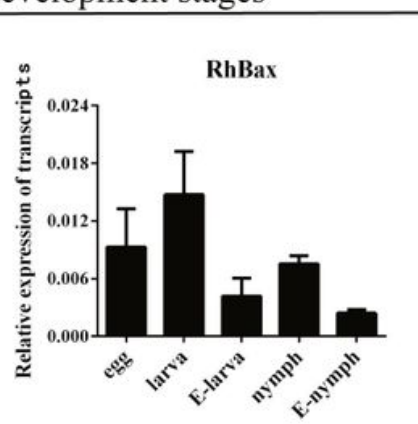

nt sex

(D) salivary gland
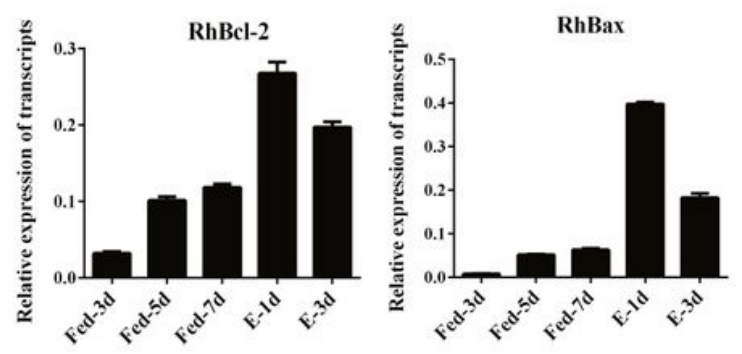
midgut
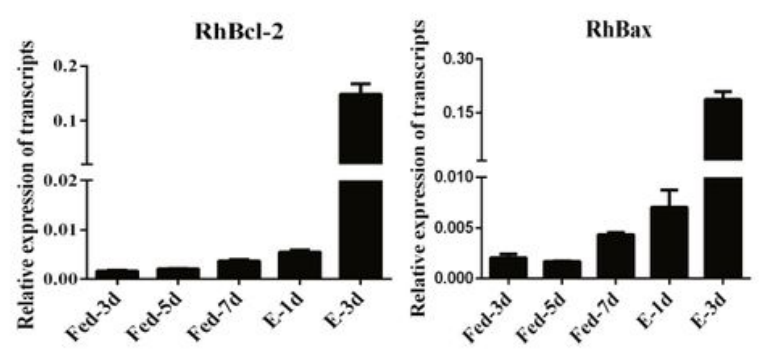

(E) ovary
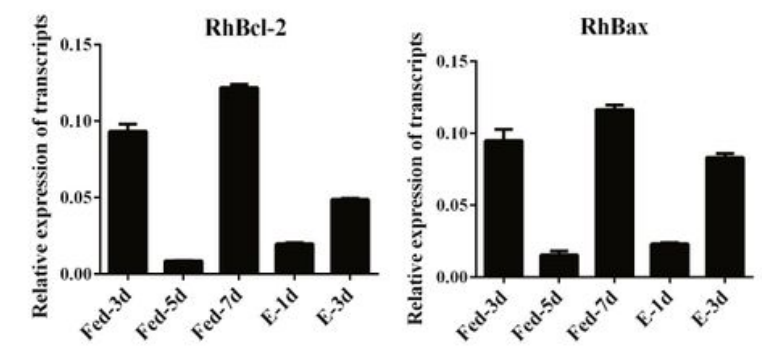

Figure 2

Transcription analysis of RhBcl-2 and RhBax in Rhipicephalus haemaphysaloides. (A) RT-qPCR analysis of RhBcl-2 and RhBax gene expression during different development periods. (B) RT-qPCR analysis of $\mathrm{RhBcl}-2$ and $\mathrm{RhBax}$ gene expression according to sex. (C-E) Transcription of RhBcl-2 and RhBax in different tissues of R. haemaphysaloides. (F: fed, E: engorged). 


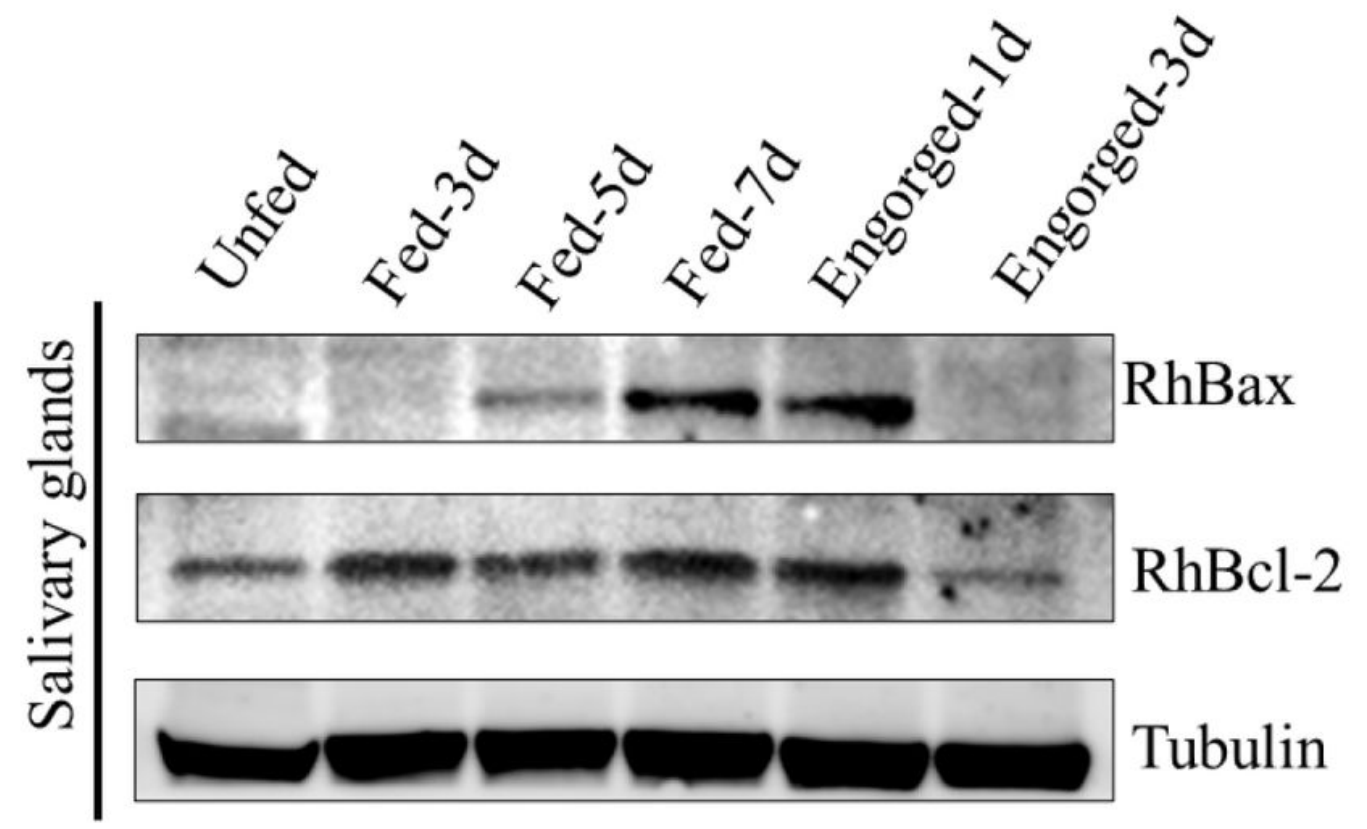

Figure 3

Western blot detection of RhBcl-2 and RhBax of R. haemaphysaloides salivary glands at different feeding times. Tick salivary glands were screened with sera anti-RhBcl-2, RhBax and anti-tubulin serum was used as control. 
(A) KDa

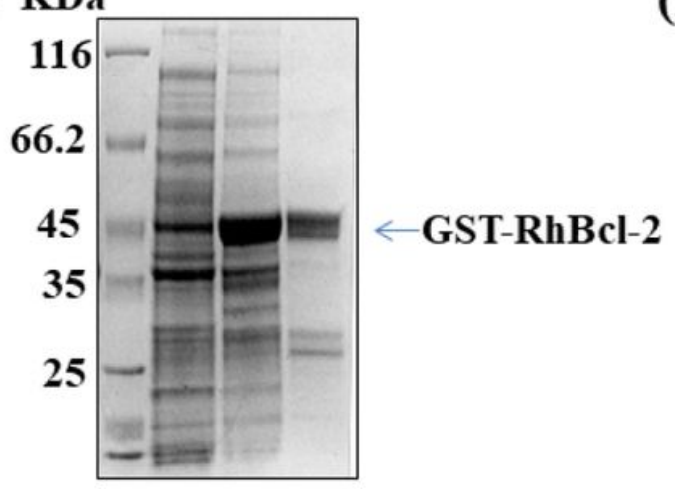

(C) KDa

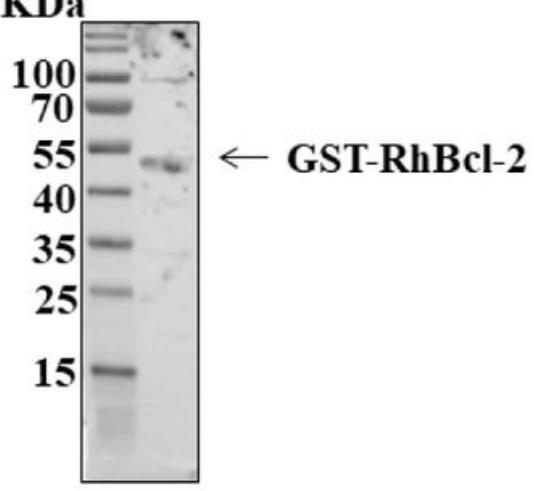

(B) KDa

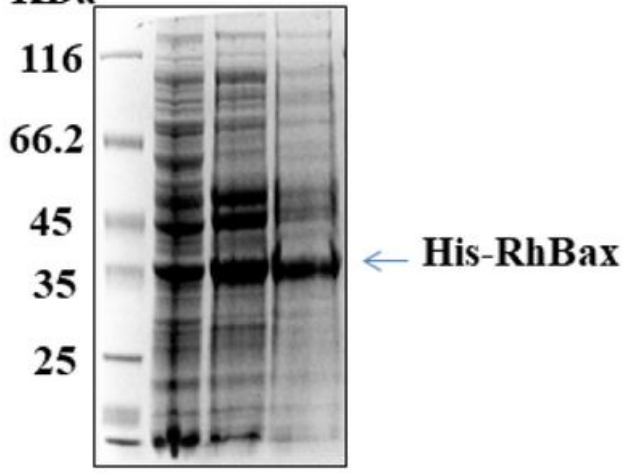

(D) KDa

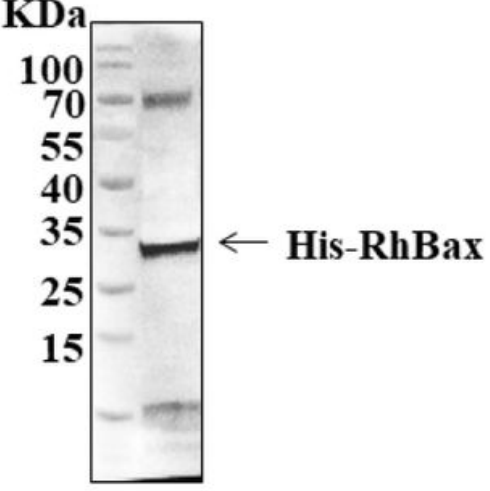

Figure 4

Expression of recombinant RhBcl-2 and RhBax. (A, B) Purification of recombinant RhBcl-2 and RhBax. Lane 1: M: the protein marker; Lane 2: non-induced; Lane 3: $1 \mathrm{mM}$ IPTG induced expression for $8 \mathrm{~h}$; Lane 4: recombinant protein after purification. (C, D) Western-Blotting detection of RhBcl-2 and RhBax. Purified $\mathrm{RhBcl}-2$ and $\mathrm{RhBax}$ were immunoblotted with the anti-sera against $\mathrm{RhBcl}-2$ and $\mathrm{RhBax}$, respectively. 
(A)

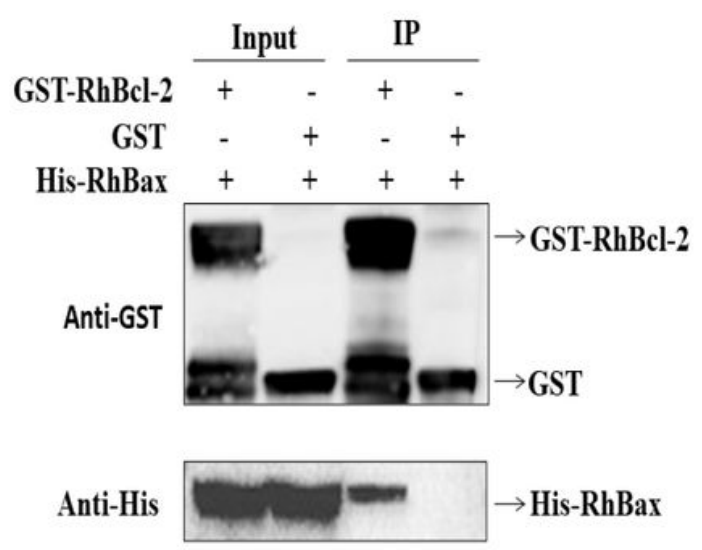

(B)

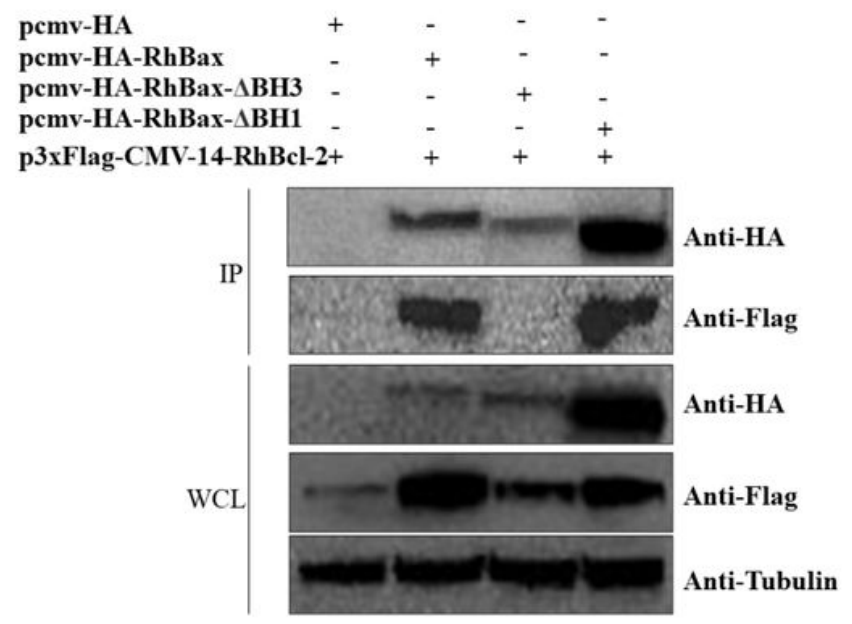

\section{Figure 5}

GST pull-down binding and co-transfection assays of the RhBcl-2 and RhBax. (A) The input protein samples showed pull-down binding assays of His-RhBax as prey with RhBcl-2 as a bait. The IP shows pull-down binding assays of His-RhBax with the GST-RhBcl-2. GST is used as a negative control. (B) Western blotting analysis of the interaction between RhBcl-2 and RhBax by co-transfection into HEK-293T cells. Lane 1: Cells transfected individually with pcmv-HA vector. Lane 2-4: Cells co-transfected p3×FlagCMV-14-RhBcl-2 with and pcmv-HA-Rhbax, pcmv-HA-Rhbax- $\triangle B H 3$ and pcmv-HA-Rhbax- $\Delta B H 1$. 
(A)

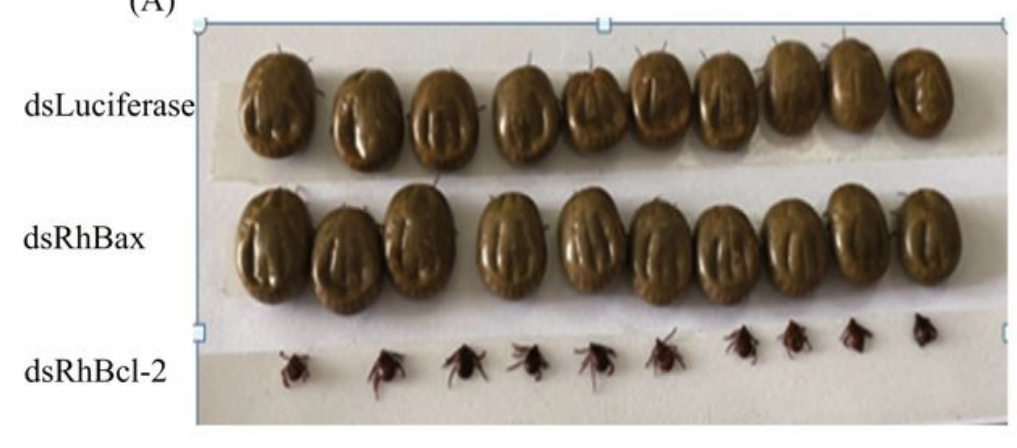

(C)
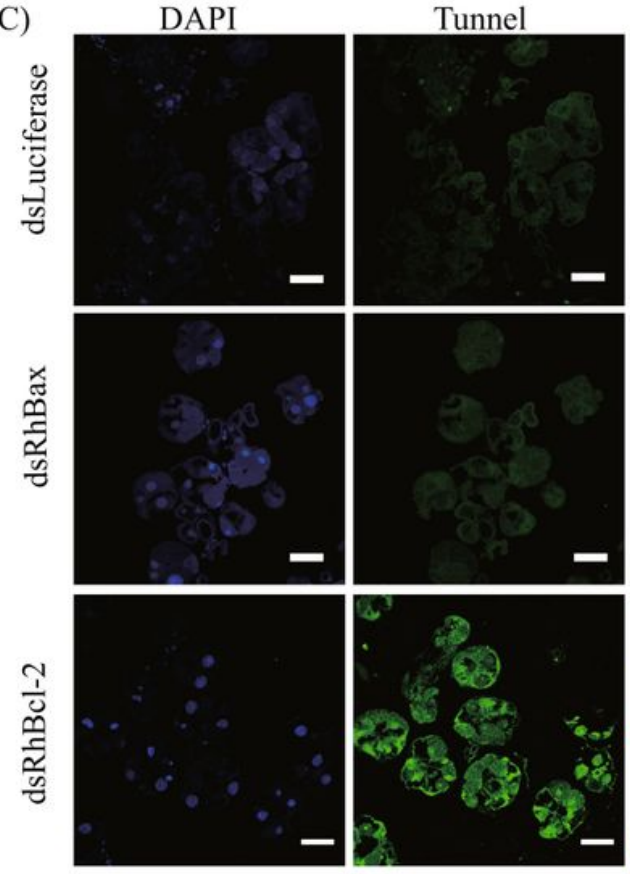

Merge

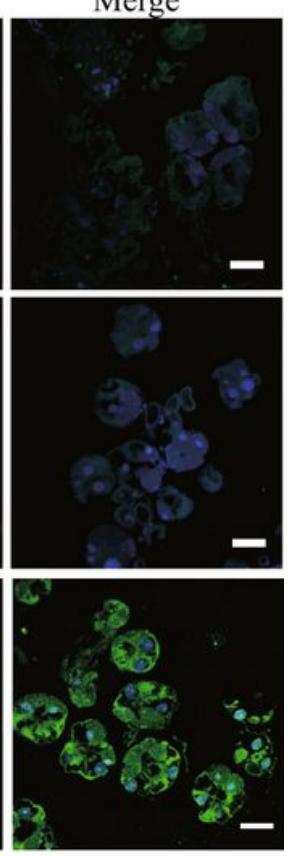

(D)

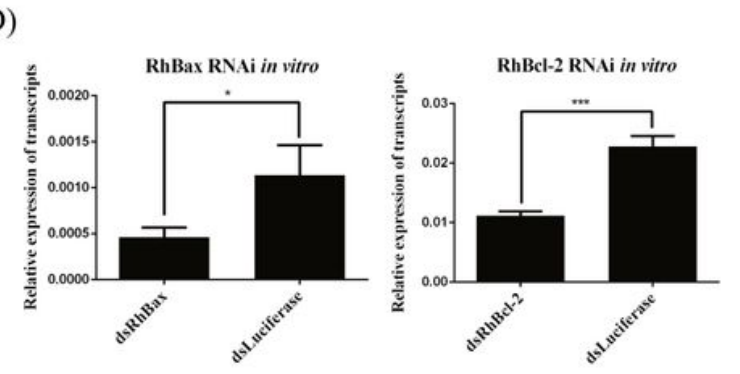

Tunnel

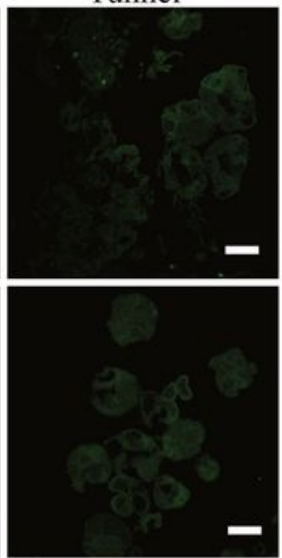

(B)

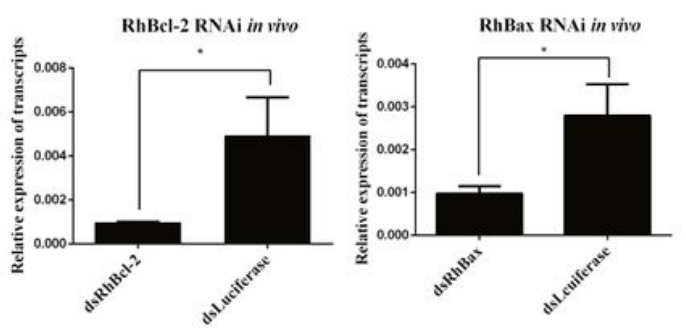

Figure 6

Biological effects of RNAi of Rhipicephalus haemaphysaloides RhBcl-2 and RhBax. (A) Images comparing ticks inoculated with ds RhBcl-2 and RhBax with control groups on day 7 of feeding. dsBcl-2 inhibited the blood-feeding process and resulted in ticks smaller than the controls. (B) Confirmation of $\mathrm{RhBcl}-2$ and RhBax silencing using RT-qPCR. Total RNA was extracted on feeding day 5 from female ticks injected with dsRNA. Bars represent mean relative expression of $\mathrm{RhBcl}-2$ and $\mathrm{RhBax}$; error bars represent standard error. ${ }^{* \star}, \mathrm{P}<0.001$, based on two-tailed Student's $t$ tests. (C) TUNEL staining assays of apoptosis levels after interfering with the salivary glands by dsRNA of RhBcl-2 and RhBax. DNA was stained with DAPI. Scale bars: $50 \mu \mathrm{m}$. (D) Confirmation of RhBcl-2 and RhBax silencing using RT-qPCR.

\section{Supplementary Files}

This is a list of supplementary files associated with this preprint. Click to download.

- GraphicalAbstract.eps

- aadditonalfile1.docx 